\title{
Prediction of overall survival time in patients with colon adenocarcinoma using DNA methylation profiling of long non-coding RNAs
}

\author{
QIONGYING ZHANG $^{1 *}$, ZHUO LIN $^{2 *}$, HAIYAN ZHANG $^{3}$, XIAODONG BAO $^{4}$ and HUXIANG ZHANG ${ }^{4}$ \\ Departments of ${ }^{1}$ Pathology, ${ }^{2}$ Hepatology, ${ }^{3}$ Emergency and ${ }^{4}$ Central Laboratory, \\ The First Affiliated Hospital of Wenzhou Medical University, Wenzhou, Zhejiang 325015, P.R. China
}

Received May 14, 2019; Accepted November 22, 2019

DOI: $10.3892 / 01.2019 .11236$

\begin{abstract}
Long non-coding RNAs (lncRNAs) are a subgroup of RNAs able to regulate gene expression at the epigenetic level, and are therefore central to the regulation of numerous biological processes and the progression of multiple cancer types. However, IncRNAs have not been identified to considerably influence overall survival (OS) outcome in numerous different types of cancer. The majority of studies investigating the association between lncRNAs and epigenetic regulation have focused on their altered expression levels in cancerous cells, and few studies have focused on determining the correlation between IncRNAs and OS time. In the present study, comprehensive lncRNA expression analysis was performed on a cohort of patients diagnosed with colon adenocarcinoma (COAD) using the least absolute shrinkage and selection operator method (LASSO). Subsequently, the construction of a prognostic methylation-based predictive system was performed based on the results of LASSO analysis. Functional enrichment analysis of lncRNA co-expression genes was also performed. According to the results of the present study, the classifier was able to significantly predict the prognosis of patients with COAD, and the investigation of the relevant elucidated genes further revealed the mechanism of COAD pathogenesis.
\end{abstract}

\section{Introduction}

Colon cancer is one of the most prevalent and severe cancer types. Due to frequent treatment failure and a high recurrence

Correspondence to: Dr Huxiang Zhang, Central Laboratory, The First Affiliated Hospital of Wenzhou Medical University, Nanbaixiang Street, Wenzhou, Zhejiang 325015, P.R. China

E-mail: 383951930@qq.com

${ }^{*}$ Contributed equally

Key words: DNA methylation, colon cancer, colon adenocarcinoma, long non-coding RNA, overall survival rate, it has been reported as the second most prevalent malignancy and the third leading cause of cancer-associated mortality worldwide. Globally, this results in $\sim 500,000$ mortalities and $\sim 1$ million new diagnoses each year $(1,2)$. Colon adenocarcinoma (COAD) is the most prevalent histological subtype of colon cancer and its incidence is increasing due to numerous factors, including genetic predisposition, obesity, and dietary and individual lifestyle choices (3-5). The current clinical treatment strategies for COAD include surgery, chemotherapy and dietary regulation (6).

Despite improvements in the diagnostic and therapeutic strategies for COAD over recent decades, disease etiology remains poorly characterized, and the prognosis for patients with COAD remains unsatisfactory. This is primarily due to a lack of clinically significant biomarkers, which may facilitate early diagnosis or enable the precise prediction of resistance to conventional treatment protocols $(7,8)$. Colon cancer exhibits a poor overall survival (OS) rate, particularly in patients with advanced or metastatic COAD, and treatment options require urgent improvement (9). Thus, the identification of novel biomarkers may improve the early diagnosis and treatment of COAD, consequently resulting in a reduction in the mortality rate.

The discovery and characterization of the function of long non-coding (lnc)RNAs (>200 nucleotides) represents an opportunity to increase understanding of the molecular mechanisms involved in cancer progression, and also provides novel potential therapeutic targets $(10,11)$. IncRNAs have been implicated in numerous cellular processes, including cell proliferation and differentiation, chromatin dynamics and gene expression (12-15). Moreover, lncRNAs have been implicated in multiple epigenetic regulatory mechanisms, and the misregulation of epigenetic markers is associated with the inappropriate activation or inhibition of various genes, contributing to cancer development and progression $(16,17)$. lncRNAs serve a critical role in the pathogenesis of numerous malignancies, including lung (18), endometrial (19), breast (20) and hepatocellular cancer (21). Numerous studies have demonstrated that the regulation of epigenetic modifications may contribute to several pathological processes involved in cancer progression. Additionally, it has been discovered that the functions of specific lncRNAs can be altered by methylation, and 
could therefore influence tumor suppressor genes and proteins, resulting in the regulation of oncogenesis and tumor development (22).

It was discovered that hypermethylation of the maternally expressed 3 (MEG3) promoter in patients with acute myeloid leukemia decreased expression of lncRNA MEG3, compared with the control group (23). Moreover, Guo et al (24) determined that the downregulation of IncRNA LOC100130476 was caused by the hypermethylation of $\mathrm{CpG}$ sites in esophageal squamous cell carcinoma (ESCC) cells, and that this was also associated with ESCC progression.

In summary, the DNA methylation patterns of specific lncRNAs may represent useful biomarkers, improving the precision of diagnosis and prognosis in patients with COAD.

In the current study, 485,577 $\mathrm{CpG}$ sites located $<2 \mathrm{~kb}$ upstream of IncRNA transcriptional start sites (TSSs) were identified, and subsequently, a comprehensive lncRNA expression analysis was performed on data from patients with COAD, in order to develop a prognostic methylation-based classifier. The least absolute shrinkage and selection operator method (LASSO) (25), and the results of the present study, indicated that the classifier was able to divide patients into different risk grades corresponding to their respective OS times. Functional enrichment analysis of 1ncRNA co-expressed genes was also investigated.

\section{Materials and methods}

Dataset retrieval and processing. Datasets comprised of DNA methylation, RNA-seq and clinical data collected from patients with COAD were downloaded from TCGA data portal (https://portal.gdc.cancer.gov/). DNA methylation profiling was performed using the Infinium HumanMethylation450 BeadChip (Illumina, Inc.), and RNA-seq was carried out using the IlluminaHiSeq RNA-seq platform (Illumina, Inc.). The lncRNA annotation file was obtained from GENCODE (https://www.gencodegenes.org/). Kyoto Encyclopedia of Genes and Genomes (KEGG) is a database that systematically analyzes the metabolic pathways of gene products in cells and the functions of the gene products. In the present study, the relative database from KEGG was obtained and analyzed using Multi-Experiment Matrix (MEM) and The Database for Annotation, Visualization and Integrated Discovery (DAVID; (https://david.ncifcrf.gov/). All datasets are publicly available and the study protocol adhered to the publication guidelines.

CpG sites of lncRNAs. DNA methylation was found to occur predominantly on cytosine, followed by guanine residues (CpG methylation). The methylation of $\mathrm{CpG}$ sites was reported as a $\beta$-value ranging from 0 (unmethylated) to 1 (completely methylated). Normalization of the methylation $\beta$-values was conducted using the 'minfi' package of R software (3.5.1). CpG sites located $<2 \mathrm{~kb}$ upstream of an lncRNA transcriptional start site (TSS) were selected from 485,577 possible CpG sites in the HumanMethylation450 BeadChip, according to the annotation from TCGA. Several differentially-methylated $\mathrm{CpG}$ sites between COAD and normal adjacent tissues (from the same patients) were also selected using the 'minfi' package. The Student's t-test was performed to compare the $\beta$-values of $\mathrm{CpG}$ sites between COAD and normal adjacent tissues. In the present study, a difference in the $\beta$-value of $\mathrm{CpG}$ sites $>1$ (between COAD and normal adjacent tissues) was considered significant, and was subsequently selected for construction of the database used for further analysis. Inhibition of multiple genes can occur when certain regions of DNA sequences are methylated (26). In order to screen the $\mathrm{CpG}$ sites that would exhibit a negative linear correlation between methylation and lncRNA expression level, correlation analysis was performed and an associated P-value was calculated.

Methylation-based classifier for the prediction od patient OS time. The association between the methylation value of specific $\mathrm{CpG}$ sites selected in the previous step and the OS times of patients was assessed using a univariate Cox regression model. Following the identification of $\mathrm{CpG}$ sites with a statistically significant association with OS, in order to develop a methylation-based classifier to predict OS, the Least Absolute Shrinkage and Selection Operator (LASSO) regression model was then constructed to identify $\mathrm{CpG}$ site predictors using estimated regression coefficients. As a result, a methylation-based classifier was constructed that predicted OS times using the fitted LASSO regression model. Lasso regression is a linear regression model that uses shrinkage in order to improve the predictive accuracy and interpretability of regression models, by altering the model fitting process to select only a subset of the provided covariates for use in the final model. Shrinkage indicates that data values have shrunk towards a central point, such as the mean (27).

Predictive and prognostic analysis of methylation-based classifiers. The fitted LASSO regression model was used to estimate patient risk scores, and the predictive accuracy of each selected classifier was evaluated via the construction of a fitted model for OS; this utilized time-dependent receiver operating characteristic (ROC) analysis, and was based on the predetermined risk scores. In order to analyze the association between certain clinicopathological characteristics and the methylation-based classifiers and OS, univariate and multivariate Cox regression analyses were employed to identify predictors. The predictive accuracy of certain clinicopathological variables, and the methylation-based classifiers, was evaluated using the area under the curve (AUC) of time-dependent ROC curves constructed via the 'timeROC' R package. High- or low-risk groups were formed according to the median cut-off point of the risk score, and Kaplan-Meier analysis was then performed to estimate and compare the OS times of patients in each group.

IncRNA co-expression gene and functional enrichment analysis. The co-expressed genes of lncRNAs were identified using MEM, a web-based, multi-experiment gene expression query and visualization tool. It retrieves information from several hundred publicly available gene expression datasets that represent different tissues, diseases and conditions. To improve compatibility and comparability, the datasets were arranged according to their platform type. Given a gene as an input, MEM ranks other genes by their similarity in each individual dataset. This is a novel rank aggregation method which identifies individual rankings to produce a score of statistically significant estimation, and hence a ranking across all datasets simultaneously. The new significance score is also 
capable of identifying a subset of datasets where genes exhibit significant similarity, thus allowing elimination of datasets in which significant correlation is missing or not detectable (27).

DAVID is a bioinformatics resource consisting of an integrated biological knowledgebase and analytical tools, facilitating the systematic extraction of biological meaning from large gene/protein lists derived from genomic studies. Function enrichment analysis of the co-expression genes was performed using DAVID Bioinformatics Resources, and the significant enrichment terms were visualized using the 'ggplot2' package (2.3.0.0) of R.

Statistical analysis. LASSO is a compression estimation model that constructs a penalty function, helping a model to compress the regression coefficients, set certain coefficients to zero and to select variables. Comparisons between ROC curves were calculated by quantifying the AUC, and the AUC of a classifier was equivalent to the probability that the classifier would rank higher at a randomly chosen positive instance, compared with a randomly chosen negative instance. Cox regression models are typically used to predict the prognosis of cancers and chronic diseases with the following formula: $\mathrm{h}(\mathrm{t} / \mathrm{X})=\mathrm{h} 0(\mathrm{t}) \exp (\beta 1 \mathrm{X} 1$ $+\beta 2 X 2+\ldots \ldots+\beta p X p), h 0(t):$ Benchmark risk function, $\mathrm{X} 1, \mathrm{X} 2 \ldots \mathrm{Xp}$ : Variable, $\beta 1, \beta 2 \ldots \beta \mathrm{p}$ : Regression coefficient. Kaplan-Meier analysis (a product-limiting method) was used to estimate OS, according to a probability theory called the multiplication rule. $\mathrm{P}<0.05$ was considered to indicate a statistically significant result.

\section{Results}

Clinical characteristics of the patient datasets. A the time of retrieval, TCGA contained records of 459 patients with COAD. However, only 293 patients had records of both DNA methylation and OS data. Table I exhibits the summary of the clinical characteristics of the 293 patients. Regarding the methylation status, there were a total of 334/459 COAD samples that provided methylation data. Of these 334, 296 samples were taken from COAD tissue and 38 samples were taken from corresponding paracancerous adjacent tissues. Regarding RNA-seq data, there were 497 tissue samples (459 COAD and 38 adjacent paracancerous tissues). A total of 314 datasets provided both methylation and RNA-seq data.

Selection of $\mathrm{Cp} G$ sites and construction of the methylation-based classifier. Following screening, a total of 11,259 CpG sites located $<2 \mathrm{~kb}$ upstream of lncRNA TSS's (excluding the $\mathrm{CpG}$ sites on the $\mathrm{X}$ and $\mathrm{Y}$ chromosome) were identified. Using the annotation of HumanMethylation450 BeadChip by TCGA and the 'minfi' package in R, 4,876 CpG sites with differential methylation between COAD and normal adjacent tissues were selected, 2,276 of which had a $\beta$-value difference $>0.1$. Among the 2,276 CpG sites, there were 1,092 whose linear correlation between the methylation and the expression levels of lncRNA were negative.

From the 1,092 aforementioned $\mathrm{CpG}$ sites, univariate Cox regression analysis identified $24 \mathrm{CpG}$ sites with a statistically significant association with OS time. In order to develop a methylation-based classifier to predict OS, the LASSO regression model was then employed using the methylation data of
Table I. Clinical characteristics of patients with colon adenocarcinoma.

\begin{tabular}{lc}
\hline Clinicopathological variables & Patients, $\mathrm{n}$ \\
\hline Age & 293 \\
$<60$ years & $98(33.4 \%)$ \\
$\geq 60$ years & $195(66.6 \%)$ \\
Sex & 293 \\
Men & $158(53.9 \%)$ \\
Women & $135(46.1 \%)$ \\
Vascular invasion & 255 \\
Present & $60(20.5 \%)$ \\
Absent & $195(66.6 \%)$ \\
KRAS mutation & 44 \\
Yes & $21(7.2 \%)$ \\
No & $23(7.8 \%)$ \\
Pathological stage & 283 \\
I + II & $157(53.6 \%)$ \\
III + IV & $126(43.0 \%)$ \\
Recurrence & $69(23.5 \%)$ \\
Death & $69(23.5 \%)$ \\
\hline
\end{tabular}

these 24 sites. The LASSO regression method was then used to determine the regression coefficient of the $17 \mathrm{CpG}$ sites, and the statistical significance was calculated. There were 4 CpG sites (cg00333800, cg19511844, $\operatorname{cg} 02908900$ and cg23152885) with a coefficient $>0$, exhibiting a positive correlation. Another $13 \mathrm{CpG}$ sites exhibited negative regression coefficients $<0$. The corresponding coefficients of the $17 \mathrm{CpG}$ sites are depicted in Fig. 1A and B, and a risk score-fitted model for methylation-based classifier was calculated using the following formula: $0.231 \mathrm{x}$ beta_cg $00333800+0.125$ $\mathrm{x}$ beta_cg02908900-0.485 x beta_cg03694713-0.076 $\mathrm{x}$ beta_cg05146399-0.120 x beta_cg05500125-0.666 $x$ beta_cg08736522-0.195 x beta_cg08866665-0.395 $\mathrm{x}$ beta_cg09133892-0.036 x beta_cg10405604-0.127 $\mathrm{x}$ beta_cg10508317-0.043 x beta_cg12967319-1.028 x beta_cg14319657-0.202 x beta_cg14858267+0.143 x beta_cg19511844+0.122 x beta_cg23152885-0.332 x beta_cg25137968-0.027 x beta_cg26186727. Table II contains information on the characteristics of the $17 \mathrm{CpG}$ sites selected using LASSO. Table III indicates the computer-generated risk score of the methylation-based classifier for a selection of patients. Comparison between COAD and normal adjacent tissues indicated that the methylation levels in $12 \mathrm{CpG}$ sites were upregulated, and downregulated in $5 \mathrm{CpG}$ sites in the cancerous tissues (Fig. S1). Additionally, unsupervised hierarchical clustering analysis suggested that the methylation data of these $17 \mathrm{CpG}$ sites were able to accurately discriminate between COAD and normal adjacent tissue samples (Fig. 1C).

Predictive and prognostic accuracy of the methylation-based classifier. A risk score for each patient was calculated according to the methylation-based classifier, and the predictive accuracy of the classifier was evaluated using a 
Table II. Characteristics of CpG sites selected by the least absolute shrinkage and selection operator model. CGI, CpG island; TSS, transcriptional start site.

\begin{tabular}{|c|c|c|c|c|c|}
\hline CG_ID & Gene symbol & CG chromosome location & $\begin{array}{c}\text { Position } \\
\text { to TSS }\end{array}$ & CGI coordinate & $\begin{array}{c}\text { Feature } \\
\text { type }\end{array}$ \\
\hline $\operatorname{cg} 00333800$ & CTD-2382H12.1 & chr18: 78927878-78927879 & TSS1500 & chr18:78925187-78925397 & S Shelf \\
\hline cg02908900 & MEOX2-AS1 & chr7: 15687196-15687197 & TSS1500 & chr7:16399497-16399700 & \\
\hline cg03694713 & RP11-175E9.1 & chr8: 23706643-23706644 & TSS 1500 & chr8:23704962-23707662 & Island \\
\hline $\operatorname{cg} 05146399$ & LINC00635 & chr3: $107883413-107883414$ & TSS 1500 & chr3:107927623-107928094 & \\
\hline $\operatorname{cg} 05500125$ & RP11-66B24.2 & chr15: 100849818-100849819 & TSS 1500 & chr15:100849527-100850055 & Island \\
\hline $\operatorname{cg} 08736522$ & RP11-108M9.3 & chr1: 16872351-16872352 & TSS 1500 & chr1:16872131-16873554 & Island \\
\hline $\operatorname{cg} 08866665$ & XXуac-YX65C7_A.3 & chr6: 169289797-169289798 & TSS1500 & chr6:169248451-169248952 & \\
\hline cg09133892 & LINC01301 & chr8: 60413320-60413321 & TSS200 & chr8:60516615-60517614 & \\
\hline $\operatorname{cg} 10405604$ & RP11-66B24.2 & chr15: 100850054-100850055 & TSS 1500 & chr15:100849527-100850055 & Island \\
\hline $\operatorname{cg} 10508317$ & RP11-806H10.4 & chr17: 78359065-78359066 & TSS 1500 & chr17:78358737-78360957 & Island \\
\hline $\operatorname{cg} 12967319$ & MEG3 & chr14: 100825660-100825661 & TSS1500 & chr14:100825706-100826372 & N Shore \\
\hline $\operatorname{cg} 14319657$ & LINC00898 & chr22: $47632172-47632173$ & TSS 1500 & chr22:47212945-47213572 & \\
\hline $\operatorname{cg} 14858267$ & RP4-555D20.3 & chr3: 43996268-43996269 & TSS 1500 & chr3:43994915-43999612 & Island \\
\hline $\operatorname{cg} 19511844$ & RP11-387H17.4 & chr17: 39927866-39927867 & TSS200 & chr17:39926973-39927799 & S Shore \\
\hline $\operatorname{cg} 23152885$ & RP11-247C2.2 & chr15: 74129941-74129942 & TSS 1500 & chr15:74127529-74130703 & Island \\
\hline $\operatorname{cg} 25137968$ & RP11-439A17.4 & chr1: $121117978-121117979$ & TSS 1500 & chr1:121118208-121118586 & N Shore \\
\hline $\operatorname{cg} 26186727$ & RP11-676J15.1 & chr18: 72867299-72867300 & TSS 1500 & chr18:72866730-72869636 & Island \\
\hline
\end{tabular}

Table III. Samples and their relative risk score computed are listed in the table.

\begin{tabular}{lc}
\hline Sample name & TCGA4NA93T01 \\
\hline cg00333800 & 0.384621 \\
$\operatorname{cg} 02908900$ & 0.726879 \\
$\operatorname{cg} 03694713$ & 0.683491 \\
$\operatorname{cg} 05146399$ & 0.927501 \\
$\operatorname{cg} 05500125$ & 0.033916 \\
$\operatorname{cg} 08736522$ & 0.574554 \\
$\operatorname{cg} 08866665$ & 0.079631 \\
$\operatorname{cg} 09133892$ & 0.919498 \\
$\operatorname{cg} 10405604$ & 0.089026 \\
$\operatorname{cg} 10508317$ & 0.629167 \\
$\operatorname{cg} 12967319$ & 0.692877 \\
$\operatorname{cg} 14319657$ & 0.711359 \\
$\operatorname{cg} 14858267$ & 0.879355 \\
$\operatorname{cg} 19511844$ & 0.153435 \\
$\operatorname{cg} 23152885$ & 0.179211 \\
$\operatorname{cg} 25137968$ & 0.514649 \\
$\operatorname{cg} 26186727$ & 0.634311 \\
Score & -2.15532 \\
\hline
\end{tabular}

time-dependent ROC curve to predict OS times at several follow-up times; AUC at 1 year (0.752; 95\% CI, 0.668-0.836), 3 years $(0.728 ; 95 \%$ CI, $0.640-0.816)$ and 5 years $(0.782$; 95\% CI, 0.691-0.874).

Classification into high- or low-risk groups was defined according to the median cut-off point of the risk score.
Kaplan-Meier analysis indicated that a high risk score indicated poorer OS [Hazard ratio (HR), 4.40; 95\% CI, 2.73-7.07; $\mathrm{P}<0.001$; Fig. 2B]. A similar association was determined following the analysis of disease-free survival (DFS) times in patients (HR, 4.04; 95\% CI, 1.62-10.09; $\mathrm{P}=0.003$; Fig. 2C, and also in patients stratified according to certain clinicopathological risk factors (including age, vascular invasion and pathological stage; Fig. 3).

In order to analyze the association between certain clinical variables and OS, univariate Cox regression analysis was performed and resulted in the following predictive scores for OS: Sex (HR, 1.66; 95\% CI, 1.02-2.70; $\mathrm{P}=0.043$ ), vascular invasion ( $\mathrm{HR}, 2.58 ; 95 \% \mathrm{CI}, 1.54-4.33 ; \mathrm{P}<0.001)$, pathological stage (HR, 2.67; 95\% CI, 1.61-4.43, P<0.001) and methylation-based classifier (HR, 4.75; 95\% CI, 2.70-8.34; $\mathrm{P}<0.001)$. Subsequently, multivariable adjustment of these variables was performed, and the results indicated that the pathological stage (HR, 2.82; 95\% CI, 1.64-4.86; P<0.001) and methylation-based classifier (HR, 4.08; 95\% CI, 2.20-7.54; $\mathrm{P}<0.001)$ were both significant predictors of OS (Table IV). Time-dependent ROC curve analysis determined that the methylation-based classifier, combined with the pathological stage, provided a more accurate prediction for OS time at 1 year (AUC, 0.789; 95\% CI, 0.710-0.869), 3 year (AUC, 0.799; 95\% CI, 0.716 -0.882) and 5 year (AUC, 0.767; 95\% CI, 0.667-0.867) in patients with COAD (Fig. 4).

Identification of lncRNA co-expression genes and functional evaluation. A total of 17 lncRNAs associated with the $\mathrm{CpG}$ sites in the methylation-based classifier were identified (Table II). Moreover, 2,835 gene co-expressed with the lncRNAs, were identified using MEM analysis. DAVID was used to perform functional enrichment analysis 
Table IV. Univariate and multivariate analyses of the methylation-based classifier for overall survival.

\begin{tabular}{lrrrrrrrr}
\hline & \multicolumn{3}{c}{ Univariate analysis } & & & \multicolumn{2}{c}{ Multivariate analysis } \\
\cline { 3 - 4 } Prognostic parameter & HR & $95 \%$ CI & & P-value & & HR & $95 \%$ CI & P-value \\
\hline Age, $\geq 60$ vs. $<60$ & 1.36 & $0.79-2.32$ & & 0.267 & & & \\
Sex, men vs. women & 1.66 & $1.02-2.70$ & & 0.043 & & & & \\
Vascular invasion, present vs. absent & 2.58 & $1.54-4.33$ & $<0.001$ & & & & \\
Pathological stage, & & & & & & & \\
III + IV vs. I + II & 2.67 & $1.61-4.43$ & $<0.001$ & & 2.82 & $1.64-4.86$ & $<0.001$ \\
Methylation-based classifier, high- vs. low-risk & 4.75 & $2.70-8.34$ & $<0.001$ & & 4.08 & $2.20-7.54$ & $<0.001$ \\
\hline
\end{tabular}

HR, hazard ratio.

A

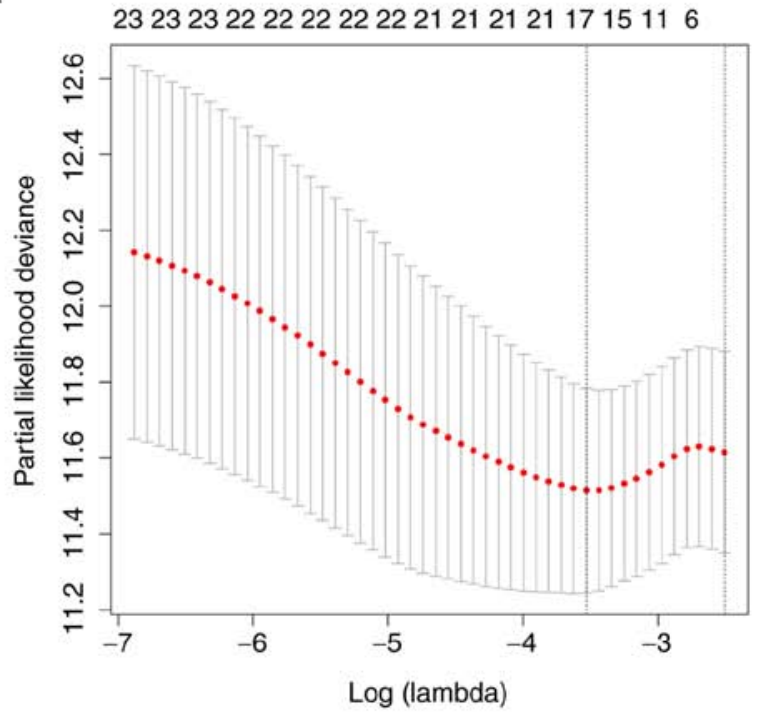

B

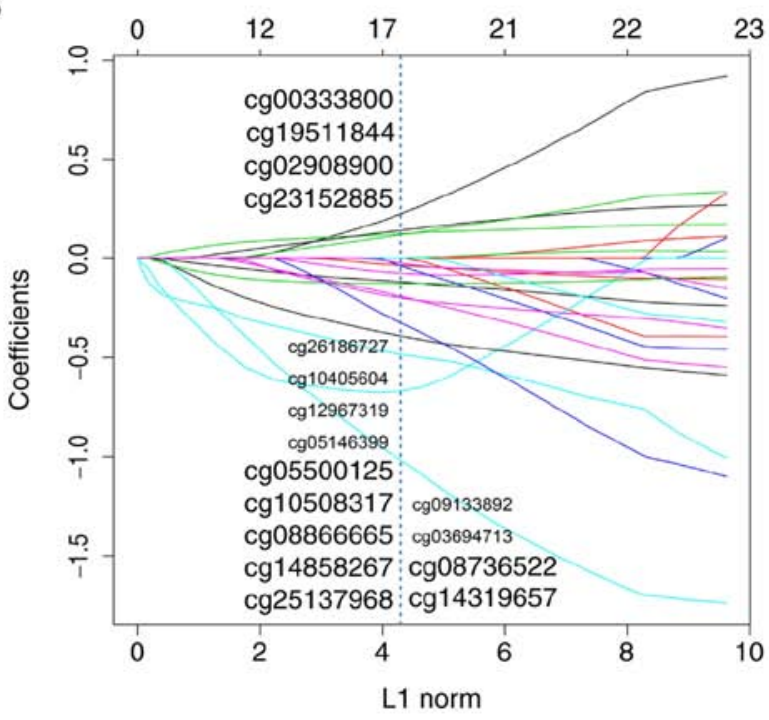

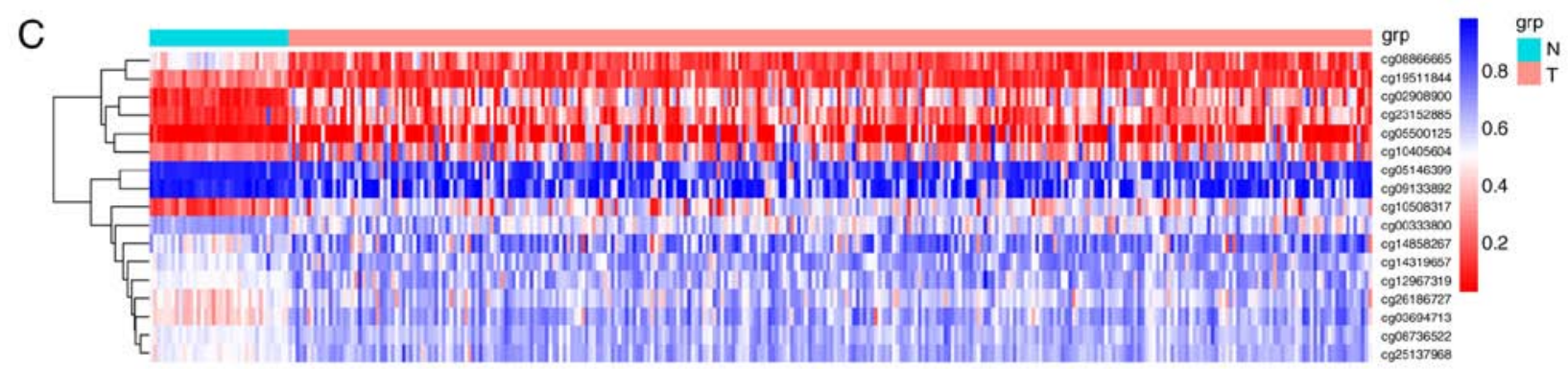

Figure 1. Construction of the methylation-based classifier. (A) Selection of CpG sites using the LASSO model. A vertical line was drawn at the value of 17 (CpG sites), and the cross-point represents the most accurate estimation. Numbers 23-1 (with one duplicate) above 17 represent the $24 \mathrm{CpG}$ sites. $\lambda$ represents variables in the LASSO regression. (B) LASSO coefficient profiles of the $17 \mathrm{CpG}$ sites. (C) Hierarchical clustering based on the differential methylation levels of the $17 \mathrm{CpG}$ sites. Dark blue represents a high proportion and dark red a low proportion. The names of the $17 \mathrm{CpG}$ sites are listed under 'grp'. Blue: N, normal samples; pink: T, tumor; $\mathrm{CpG}, \mathrm{CpG}$ island; LASSO, least absolute shrinkage and selection operator.

of the co-expressed genes, and the results indicated that the significantly enriched Gene Ontology (GO) terms were 'extracellular matrix organization' (biological process), 'cell junction' (cellular component) and 'transcriptional activator activity' (molecular function). Certain KEGG pathways were also significantly enriched, including 'MAPK-signaling pathway', 'cAMP-signaling pathway' and 'calcium-signaling pathway' (Fig. 5).

\section{Discussion}

Colon cancer is the most prevalent gastrointestinal cancer type and exhibits high incidence and mortality rates; COAD is a colon cancer subtype that accounts for $\sim 98 \%$ of new diagnoses (28). Despite advances in the diagnosis and treatment of colon cancer, due to the recognition of various prognostic and predictive factors (including age, tumor grade and stage, 

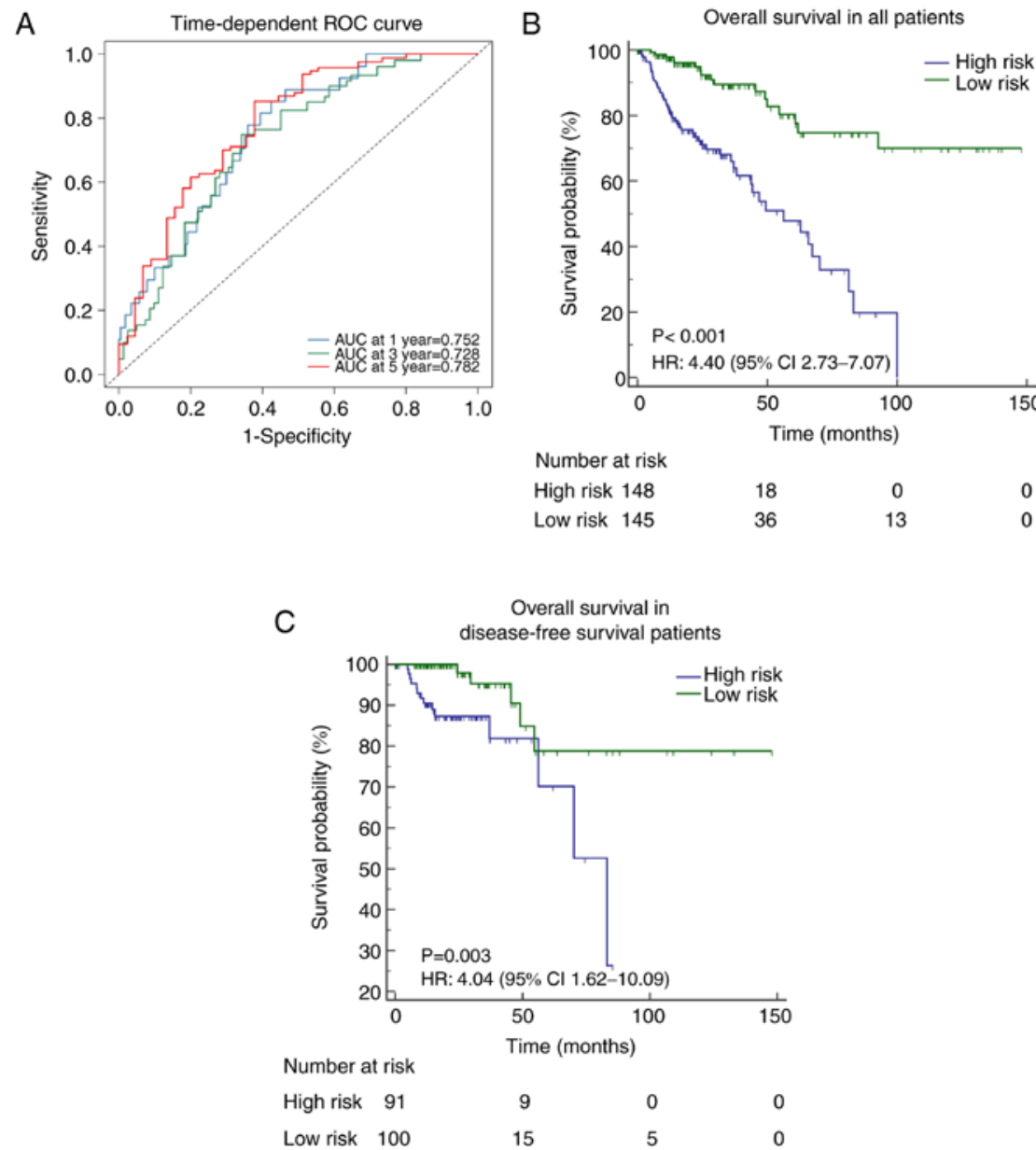

Figure 2. Time-dependent ROC curves and Kaplan-Meier survival analysis of the methylation-based classifier for OS. (A) Time-dependent ROC curves at 1 , 3 and 5 years to assess predictive accuracy for OS. (B) Kaplan-Meier analysis of OS time in all patients. (C) Kaplan-Meier analysis of OS in DFS patients. High- and low-risk groups of methylation-based classifier was calculated according to the cut-off value. ROC, receiver operating characteristic; OS, overall survival; DFS, disease-free survival; HR, hazard ratio; AUC, area under curve.

A

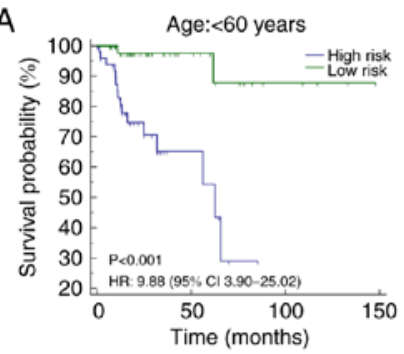

Number at risk High risk 52 Low risk 46

D

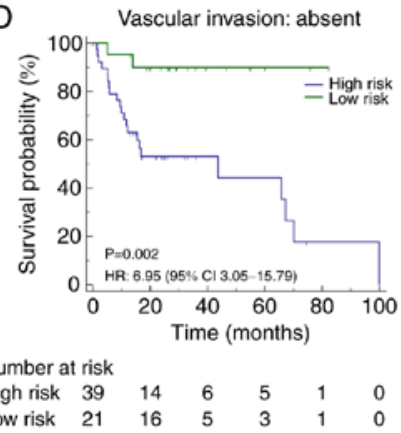

B

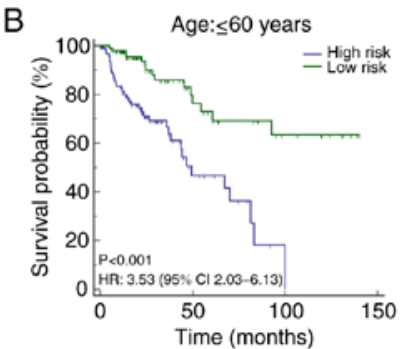

Number at risk High risk 96 Low risk 99

E

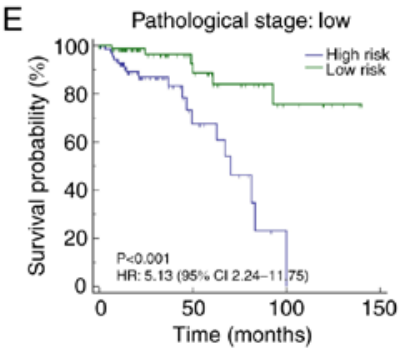

Number at risk High risk 73 Low risk 84

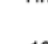

12
23
C

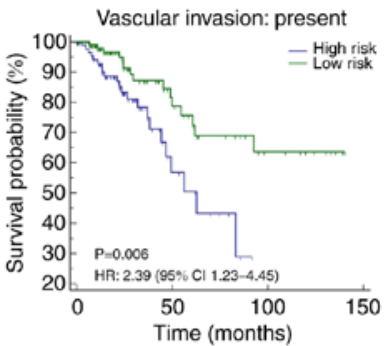

Number at risk

Number at risk

Low risk 104
$\mathrm{F}$

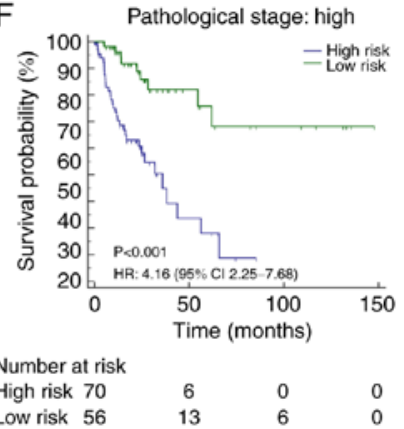

Figure 3. Kaplan-Meier survival analysis according to the methylation-based classifier stratified by clinicopathological risk factors. (A) Age $<60$; (B) age, $\geq 60$. Vascular invasion (C) present; and D) absent. Pathological stage (E) low; and (F) high. High- and low-risk groups of the methylation-based classifier were calculated according to the cut-off value. HR, hazard ratio. 
A
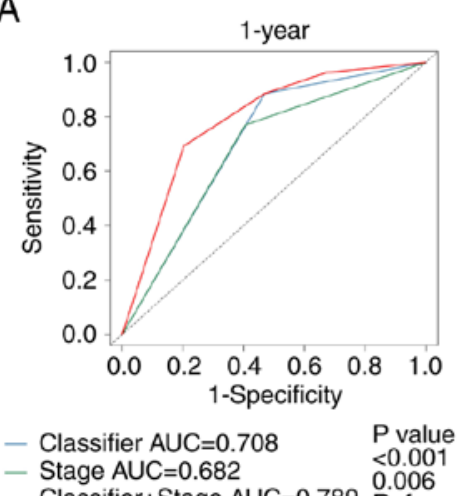

B

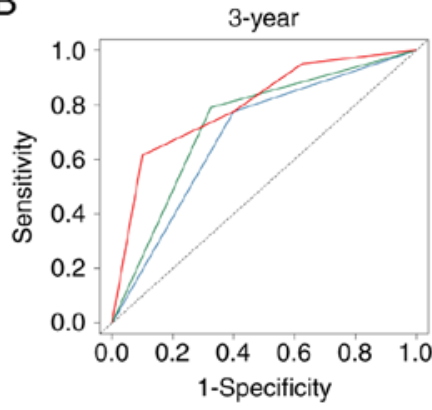

$\begin{array}{ll}\text { - Classifier AUC }=0.688 & P \text { value } \\ \text { - Stage AUC }=0.733 & <0.001\end{array}$

- Classifier+Stage AUC=0.799 $\begin{aligned} & 0.141 \\ & \text { Reference }\end{aligned}$
C
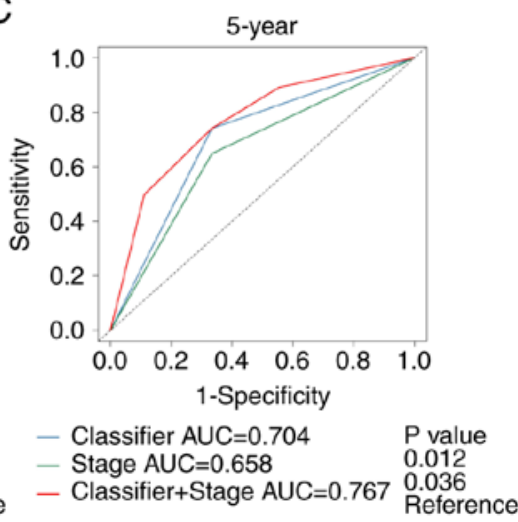

Figure 4. Time-dependent receiver operating characteristic curves compare the prognostic accuracy of the methylation-based classifier with clinicopathological risk factors. Overall survival was calculated at (A) 1-, (B) 3- and (C) 5 years. AUC, area under the curve.

A

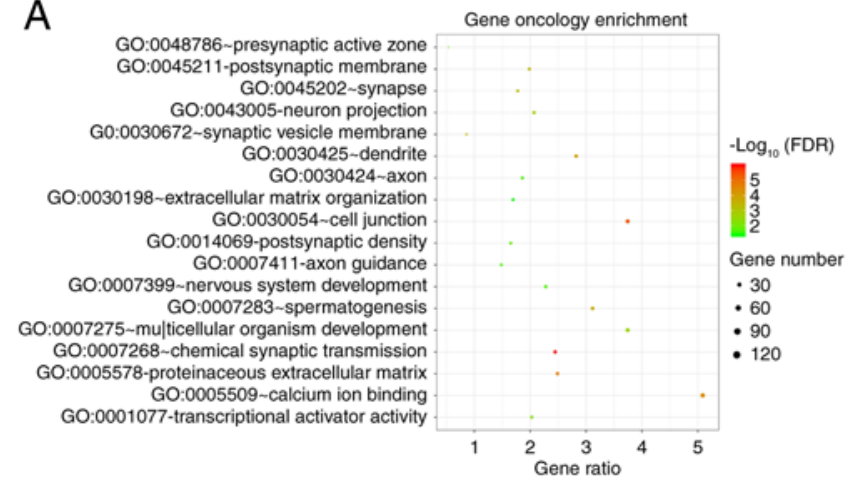

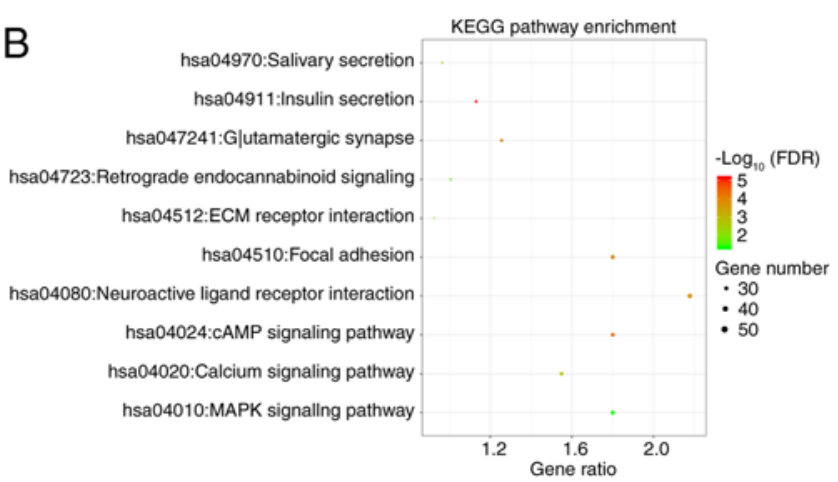

Figure 5. Functional enrichment analysis of lncRNA co-expression genes. (A) GO and (B) KEGG pathway enrichment analyses. lncRNA, long non-coding RNA; GO, gene ontology; KEGG, Kyoto Encyclopedia of Genes and Genomes.

surgical margins and the number of affected local lymph nodes), the prognosis for patients is still poor due to limited characterization of the molecular mechanism regulating COAD development and progression. Therefore, in order to develop novel and effective therapeutic approaches, comprehensive research into the molecular mechanism of COAD tumorigenesis is imperative. Although credible biomarkers for the treatment and prognosis of COAD have been characterized, the majority focus on the expression and mechanistic roles of mRNAs, lncRNAs and microRNAs (29-31). The importance of the DNA methylation profile of IncRNAs has not been fully investigated, but it shows potential to be a key novel biomarker, able to improve the OS of patients with COAD.

In the current study, comprehensive analysis of the DNA methylation profile of 1 ncRNAs was performed to investigate a large cohort of COAD samples retrieved from TCGA, with all samples showing altered DNA methylation patterns. Moreover, it was discovered that numerous $\mathrm{CpG}$ sites exhibited significantly different methylation statuses in COAD tissues, compared with adjacent normal tissues; furthermore, $24 \mathrm{CpG}$ sites were significantly correlated with OS. In accordance with LASSO regression analysis, $17 \mathrm{CpG}$ sites were identified as having statistically significant estimated regression coefficients, and their gene symbols are denoted as: CTD-2382H12.1, MEOX2-AS1, RP11-175E9.1, LINC00635, RP11-66B24.2, RP11-108M9.3, XXуас-YX65C7_A.3,
LINC01301, RP11-66B24.2, RP11-806H10.4, MEG3, LINC00898, RP4-555D20.3, RP11-387H17.4, RP11-247C2.2, RP11-439A17.4 and RP11-676J15.1. All CpG sites identified in the present study were able to significantly predict the OS times of patients with COAD. Notably, none of the aforementioned lncRNAs had been identified in previous studies.

Clinically, the methylation-based classifier constructed in the present study could be employed as a diagnostic tool, predicting OS times in patients with COAD. It was able to predict OS time and produce high- or low-risk scores for both patients with COAD and those in the DFS group. Moreover, significant predictive accuracy was exhibited at several time points during the follow-up period (according to ROC analysis), suggesting the potential to improve and individualize clinical decision-making regarding treatment programs. The analysis and assessment of COAD prognosis typically includes previously reported risk factors, including age, sex and disease stage. However, multivariate Cox regression analysis indicated that the OS time in patients with COAD could also be predicted using the methylation-based classifier as the measured parameter, further supporting the significance of methylation in disease progression. Moreover, the accuracy OS-time prediction during the follow-up period may be improved if the results of the methylation-based classifier were integrated with other clinicopathological risk factors. 
Epigenetic alterations were determined to regulate the tumorigenesis and progression of COAD. It was a complex and intricate process, but the methylation of lncRNAs is likely to be the access point to a more thorough understanding of the molecular mechanism of COAD. In order to further investigate the effects of epigenetic alterations on biological processes and pathways, comprehensive analysis of lncRNA methylation was conducted. A total of 2,899 genes were found to be co-expressed with aberrant methylation of lncRNAs, and the majority are located in pivotal cancer-signaling pathways, indicating their potential to influence tumor biology.

A limitation of the present study was the failure to elucidate the causality between the aberrant methylation patterns of lncRNAs and the occurrence of tumors. Further understanding of this mechanism may help to identify novel therapeutic targets and improve the prognosis of patients with COAD.

In conclusion, the present study identified a methylation-based classifier consisting of lncRNAs closely related to the OS times of patients with COAD, and subsequently determined that the prognosis of COAD could be accurately predicted using altered DNA methylation patterns, as well as the involvement of relevant genes in pivotal signaling pathways related to oncogenesis. An advantage of the present study was that the experiments were performed using a large population size and a sufficient data source. Additionally, the findings indicated both satisfactory independent prognostic value and biological relevant pathways. To the best of our knowledge, this was the first study to quantify the significance of the association between regulation of DNA methylation patterns by lncRNAs and the prognosis of patients with COAD.

\section{Acknowledgements}

Not applicable.

\section{Funding}

No funding was received.

\section{Availability of data and materials}

The datasets used and/or analyzed during the current study are available from the corresponding author on reasonable request.

\section{Authors' contributions}

Conceptualization of the study was performed by QZ, ZL and HXZ. ZL, QZ, HYZ and XB contributed the study methodology, resources and formal analysis. Software use and initial investigation was conducted by ZL and QZ. QZ and XB wrote the first draft, and QZ, ZL, XB, HXZ and HYZ reviewed and edited the manuscript. HXZ, ZL and QZ supervised the project. Project administration was performed by HXZ. All authors read and approved the final manuscript.

\section{Ethics approval and consent to participate}

Not applicable.

\section{Patient consent for publication}

Not applicable.

\section{Competing interests}

The authors declare that they have no competing interests.

\section{References}

1. Siegel R, Naishadham D and Jemal A: Cancer statistics, 2013. CA Cancer J Clin 63: 11-30, 2013.

2. Robertson JH, Sarkar S, Yang SY, Seifalian AM and Winslet MC: In vivo models for early development of colorectal liver metastasis. Int J Exp Pathol 89: 1-12, 2008.

3. Alaiyan B, Ilyayev N, Stojadinovic A, Izadjoo M, Roistacher M, Pavlov V, Tzivin V, Halle D, Pan H, Trink B, et al: Differential expression of colon cancer associated transcript1 (CCAT1) along the colonic adenoma-carcinoma sequence. BMC Cancer 13: 196, 2013.

4. Liu K, Yao H, Wen $Y$, Zhao $H$, Zhou N, Lei $S$ and Xiong L: Functional role of a long non-coding RNA LIFR-AS1/miR-29a/TNFAIP3 axis in colorectal cancer resistance to pohotodynamic therapy. Biochim Biophys Acta Mol Basis Dis 1864: 2871-2880, 2018.

5. Olthof MR, Hollman PC and Katan MB: Chlorogenic acid and caffeic acid are absorbed in humans. J Nutr 131: 66-71, 2001.

6. Hong J, Lu H, Meng X, Ryu JH, Hara Y and Yang CS: Stability, cellular uptake, biotransformation, and efflux of tea polyphenol (-)-epigallocatechin-3-gallate in HT-29 human colon adenocarcinoma cells. Cancer Res 62: 7241-7246, 2002.

7. Tsukuda K, Tanino M, Soga H, Shimizu N and Shimizu K: A novel activating mutation of the K-ras gene in human primary colon adenocarcinoma. Biochem Biophys Res Commun 278: 653-658, 2000.

8. Yue B, Qiu S, Zhao S, Liu C, Zhang D, Yu F, Peng Z and Yan D: lncRNA-ATB mediated E-cadherin repression promotes the progression of colon cancer and predicts poor prognosis. J Gastroenterol Hepatol 31: 595-603, 2016.

9. Benson AB, Venook AP, Al-Hawary MM, Cederquist L, Chen YJ, Ciombor KK, Cohen S, Cooper HS, Deming D, Engstrom PF, et al: NCCN guidelines insights: Colon cancer, version 2.2018. J Natl Compr Canc Netw 16: 359-369, 2018.

10. Bernard D, Prasanth KV, Tripathi V, Colasse S, Nakamura T, Xuan Z, Zhang MQ, Sedel F, Jourdren L, Coulpier F, et al: A long nuclear-retained non-coding RNA regulates synaptogenesis by modulating gene expression. EMBO J 29: 3082-3093, 2010.

11. Ponting CP, Oliver PL and Reik W: Evolution and functions of long noncoding RNAs. Cell 136: 629-641, 2009.

12. Zhu P, Wang Y, Wu J, Huang G, Liu B, Ye B, Du Y, Gao G, Tian Y, He L and Fan Z: lncBRM initiates YAP1 signalling activation to drive self-renewal of liver cancer stem cells. Nat Commun 7: 13608, 2016.

13. Fatica A and Bozzoni I: Long non-coding RNAs: New players in cell differentiation and development. Nat Rev Genet 15: 7-21, 2014.

14. He Y, Meng XM, Huang C, Wu BM, Zhang L, Lv XW and Li J: Long noncoding RNAs: Novel insights into hepatocelluar carcinoma. Cancer Lett 44: 20-27, 2013.

15. Huang JL, Zheng L, Hu YW and Wang Q: Characteristics of long non-coding RNA and its relation to hepatocellular carcinoma. Carcinogenesis 35: 507-514, 2014.

16. Lee JT: Epigenetic regulation by long noncoding RNAs. Science 338: 1435-1439, 2012.

17. Timp W and Feinberg AP: Cancer as a dysregulated epigenome allowing cellular growth advantage at the expense of the host. Nat Rev Cancer 13: 497-510, 2013.

18. Wu D, Yang B, Chen J, Xiong H, Li Y, Pan Z, Cao Y, Chen J, Li T, Zhou S, et al: Upregulation of long non-coding RNA RAB1A-2 induces FGF1 expression worsening lung cancer prognosis. Cancer Lett 438: 116-125, 2018.

19. Smolle MA, Bullock MD, Ling H, Pichler M and Haybaeck J: Long non-coding RNAs in endometrial carcinoma. Int J Mol Sci 16: 26463-26472, 2013.

20. Kim J, Piao HL, Kim BJ, Yao F, Han Z, Wang Y, Xiao Z, Siverly AN, Lawhon SE, Ton BN, et al: Long noncoding RNA MALAT1 suppresses breast cancer metastasis. Nat Genet 50: $1705-1715,2018$. 
21. Huang Y, Xiang B, Liu Y, Wang Y and Kan H: lncRNA CDKN2B-AS1 promotes tumor growth and metastasis of human hepatocellular carcinoma by targeting let-7c-5p/NAP1L1 axis. Cancer Lett 437: 56-66, 2018.

22. Yao H, Duan M, Lin L, Wu C, Fu X, Wang H, Guo L, Chen W, Huang L, Liu D, et al: TET2 and MEG3 promoter methylation is associated with acute myeloid leukemia in a hainan population. Oncotarget 8: 18337-18347, 2017.

23. Dawson MA and Kouzarides T: Cancer epigenetics: From mechanism to therapy. Cell 150: 12-27, 2012.

24. Guo W, Dong Z, Shi Y, Liu S, Liang J, Guo Y, Guo X, Shen S and Shan B: Aberrant methylation-mediated downregulation of long noncoding RNA LOC100130476 correlates with malignant progression of esophageal squamous cell carcinoma. Dig Liver Dis 48: 961-969, 2016.

25. Ranstam J and Cook JA: LASSO regression. Br J Surg 105: 1348, 2018.

26. Kulis M and Esteller M: DNA methylation and cancer. Adv Genet 70: 27-56, 2010.

27. Adler P, Kolde R, Kull M, Tkachenko A, Peterson H, Reimand J and Vilo J: Mining for coexpression across hundreds of datasets using novel rank aggregation and visualization methods. Genome Biol 2009: R139, 2009.
28. Xue W, Li J, Wang F, Han P, Liu Y and Cui B: A long non-coding RNA expression signature to predict survival of patients with colon adenocarcinoma. Oncotarget 8: 101298-101308, 2017.

29. Wang WJ, Li HT, Yu JP, Han XP, Xu ZP, Li YM, Jiao ZY and Liu HB: A competing endogenous RNA network reveals novel potential lncRNA, miRNA, and mRNA biomarkers in the prognosis of human colon adenocarcinoma. J Surg Res 235: 22-33, 2019.

30. Wang JY, Wang CL, Wang XM and Liu FJ: Comprehensive analysis of microRNA/mRNA signature in colon adenocarcinoma. Eur Rev Med Pharmacol Sci 21: 2114-2129, 2017.

31. Chen F, Li Z and Zhou H: Identification of prognostic miRNA biomarkers for predicting overall survival of colonadenocarcinoma and bioinformatics analysis: A study based on the cancer genome atlas database. J Cell Biochem 120: 9839-9849, 2019

(i) (9) This work is licensed under a Creative Commons Attribution-NonCommercial-NoDerivatives 4.0 International (CC BY-NC-ND 4.0) License. 